\title{
RESEARCH ON THE APPLICATION OF RAPID SURVEYING AND MAPPING FOR LARGE SCARE TOPOGRAPHIC MAP BY UAV AERIAL PHOTOGRAPHY SYSTEM
}

\author{
Zhiguo Gao a, Yang Song ${ }^{\text {a }}$, Changhui $\mathrm{Li}^{\text {a }}$, Fanyang Zeng ${ }^{\text {a }}$, Feng Wang ${ }^{\text {a }}$
}

(a. Guangzhou Urban Planning Design \& Survey Research Institute, No.10 jianshedama road 510060 Guangzhou city, guangdong province, China, gzg201@gzpi.com.cn)

KEY WORDS: Unmanned Aerial Vehicle, Aerial Photogrammetry System, Rapid Surveying, DOM, DLG, Large Scale Topographic Map, Low-altitude

\begin{abstract}
:
Rapid acquisition and processing method of large scale topographic map data, which relies on the Unmanned Aerial Vehicle (UAV) low-altitude aerial photogrammetry system, is studied in this paper, elaborating the main work flow. Key technologies of UAV photograph mapping is also studied, developing a rapid mapping system based on electronic plate mapping system, thus changing the traditional mapping mode and greatly improving the efficiency of the mapping. Production test and achievement precision evaluation of Digital Orth photo Map (DOM), Digital Line Graphic (DLG) and other digital production were carried out combined with the city basic topographic map update project, which provides a new techniques for large scale rapid surveying and has obvious technical advantage and good application prospect.
\end{abstract}

\section{INTRODUCTION}

\subsection{General Instructions}

In recent years, with the rapid development of China's economic, the construction of intelligent city ,digital city and meticulous management have gradually promoted to a new level, large scale topographic maps play an increasingly important role in many areas such as urban planning, construction, transportation, management, social and public services, and sustainable development ${ }^{[1-2]}$. UAV low-altitude remote sensing technology is a new direction in the field of remote sensing, with characteristics such as low cost, fast, flexible, and high efficiency, high resolution, and has been gradually applied to large scale topographic map aerial photography Measurement field ${ }^{[3-5]}$. By virtue of its own technological advantages, high-precision, high-resolution image data can be quickly and efficiently obtained, making it becoming an effective means of traditional aerial photography measurement ${ }^{[6-8]}$.

As the UAV is small size, light weight, low-altitude, the flight is extremely vulnerable to external environmental factors, resulting in flight attitude instability, poor data quality. As $\mathrm{UAV}$ aerial system configuration is generally non-Measuring digital camera with small focal length and the image, large image distortion, it brings great difficulties for the late image stitching, empty three encryption ${ }^{[9-10]}$. In this paper, the Trimble UX5HP UAV aerial system is used as a large-scale topographic map aerial survey equipment, being supported by the corresponding Aerial Imaging software and INPHO software for route design, ground monitoring, Aerial triangulation, Orth photo-mosaic, DOM production. Finally, we use our basic mapping platform to carry out the two development of the three-dimensional mapping software for image mapping, and the accuracy of topographic map is verified and analyzed.

\subsection{UX5HP Aerial Photogrammetry System}

UX5HP low-altitude UAV aerial Photogrammetry system has the characteristics of simple operation, automatic and high precision. The main technical parameters are as follows.
Table.1 main technical parameters of UX5HP

\begin{tabular}{|c|c|}
\hline Parameter list & Trimble UX5HP \\
\hline Wingspan & $100 \mathrm{~cm}$ \\
\hline weight & $2.9 \mathrm{~kg}$ \\
\hline Sensor & Sony A7@ 36MP \\
\hline battery & $14.8 \mathrm{~V}, 6600 \mathrm{MAH}$ \\
\hline MAX Flight time & $40 \mathrm{minutes}$ \\
\hline Cruise speed & $80 \mathrm{~km} / \mathrm{h}$ \\
\hline Wind resistance & $10 \mathrm{~m} / \mathrm{s}$ \\
\hline $\begin{array}{c}\text { Ground control point } \\
\text { required }\end{array}$ & PPK \\
\hline operation & automatic \\
\hline Radio link range & $5 \mathrm{~km}$ \\
\hline \\
\hline
\end{tabular}

Fig.1 UX5HP UAV

\subsection{Project Introduction}

\section{AERIAL PHOTOGRAPHY}

The 2016 urban basic topographic map update project is to meet the needs of planning $\&$ construction, realize the basic coverage of the topographic map, and establish the basic geographic information database of Guangzhou City. That includes 1: 500 digital topographic map measurement and database storage with an area of 121.4 square kilometers.

We chose 2 square kilometers as the test area which is dominated by natural village, plant, mountains, farmland and fish ponds. 
Before the surveying, the basic data of the survey area, such as basic topographic map, satellite image data, should be collected. And the flight platform equipment for inspection and routine inspection, battery and camera charging, ground control system inspection, flight test must be done to ensure the main technical parameters of the aerial system platform meet the regulatory requirements. Confirm the site reconnaissance flight area to insure there were no effects on aerial flight safety of high buildings or landscape. Look for safe landing points for takeoff and landing of UAV.

\subsection{Control Survey}

The control points of this Project are arranged according to the relevant specification requirements. The odd route is added with a flat height control point every 4-6 baselines, and the even points are added appropriately. Coordinate of the control point is measured by the continuous operation of satellite positioning service system (**CORS), which can be converted into local coordinate system coordinates with accuracy of plane level $\pm 2 \mathrm{~cm}$ and height $\pm 3 \mathrm{~cm}$. The project sets up a total of 50 control points and checkpoints.

\subsection{Route Planning}

UX5HP flight route design is accomplished by using Aerial Imaging software so as to meet the accuracy requirements of the mapping. The aerial photography is equipped with a7R $35 \mathrm{~mm}$ lens with the designed image pixels of $5 \mathrm{~cm}$. The relative flight height is designed as 375 meters with $80 \%$ heading overlap, $80 \%$ overlap.

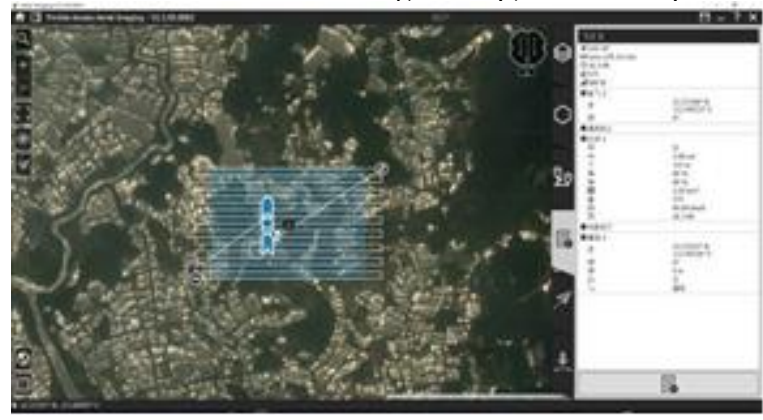

Fig.2 route planning and parameter setting

\subsection{Photograph Acquisition}

After the route planning, the flight plan has been transmitted to the UAV aerial Photogrammetry system in real-time through radio station. And after checking the UAV system, the aerial photography flight should be implemented. After the UX5HP UAV is powered on, check the device status through the ground station software. After the completion of the corresponding inspection in accordance with the operation process, start the host. Take off the UX5HP UAV through launching cradle. Monitor the aircraft status through the ground station monitoring program on the tablet, and when the flight is completed, the UAV will be landing automatically.

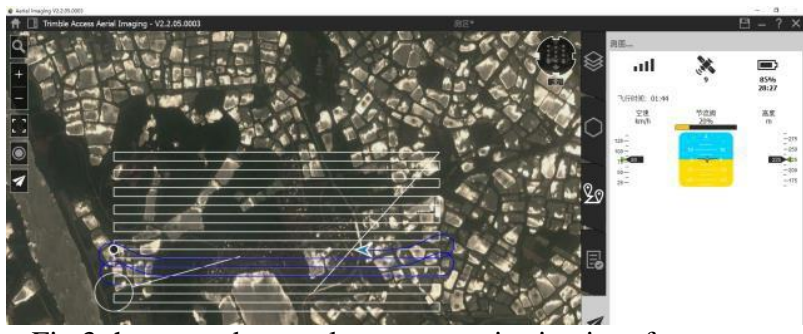

Fig.3 the ground control system monitoring interface

After aerial photography flight is completed, the data should be checked on site to see whether the number of photographs and flight path parameters are consistent and whether there exist missed images. The photo quality should also be inspected to ensure there is no ambiguity. After the inspection, if there is a quality problem, the flight should be refiled to obtain new photos. And if there has no quality problems, this aerial photography flight completed.

\section{DATA PROCESSING}

Data pre-processing mainly includes the original image inspection, POS data collation, control point data collation etc.

\subsection{Aerial triangulation}

After the pre-processing is completed, the INPHO software is used for Aerial triangulation. During Aerial triangulation process, the control points are taken at the point of the stereo image pair, and 40 points are selected as the control points from the 50 control points.

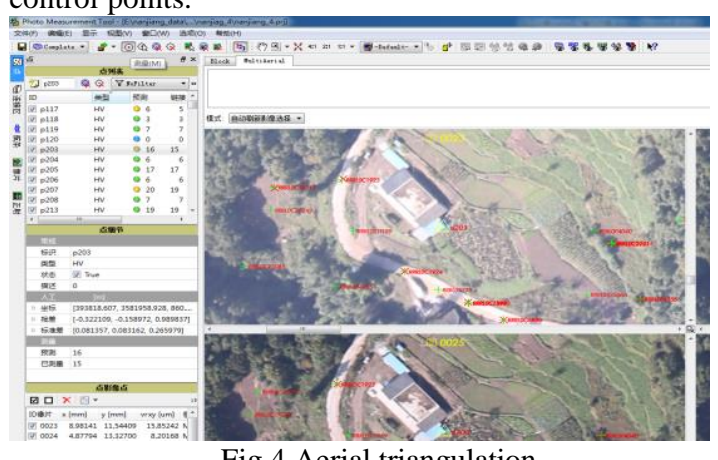

Fig.4 Aerial triangulation

The remaining points are used as checkpoints. In order to increase the intensity of model connection and avoid a global negative image caused by the big error of a certain control point, some model connection point tie points should be added to the Aerial triangulation. After repeated point adjustment and optimization, the absolute orientation accuracy of the plane $0.07 \mathrm{~m}$ and elevation $0.06 \mathrm{~m}$ are obtained ultimately.

Tab.2 Aerial triangulation accuracy

\begin{tabular}{|c|c|c|c|}
\hline & $\mathrm{X}(\mathrm{m})$ & $\mathrm{Y}(\mathrm{m})$ & $\mathrm{Z}(\mathrm{m})$ \\
\hline RMS & 0.072 & 0.067 & 0.065 \\
\hline MZX & 0.339 & 0.225 & 0.204 \\
\hline MIN & 0.003 & 0.000 & 0.000 \\
\hline point & 36 & 36 & 36 \\
\hline
\end{tabular}




\subsection{Orth photo production}

According to the results of the Aerial triangulation, the digital differential correction and image resampling of the image are obtained by Digital Elevation Modal (DEM) data, and then the DOM is generated. As the parallax of objects with the same name is big due to the low-altitude flight of the UAV, the projection error of image edge is larger according to the imaging principle of the centre of the camera, resulting in the distorted seams and building edges. Therefore, it is necessary to edit the mosaic lines of the ortho photo, and the selection and modification of the mosaic lines should avoid passing through large buildings, choose the less textured positions, stay away from the edge of the image, and follow the edge of the road and ground entities as far as possible. At the same time, the differences in color and brightness between the photos with different shooting angles should be carried out by uniform light color processing and peripheral feather processing of mosaic lines to ensure that the photos are inlaid naturally, and the overall differences of image brightness and color are consistent.

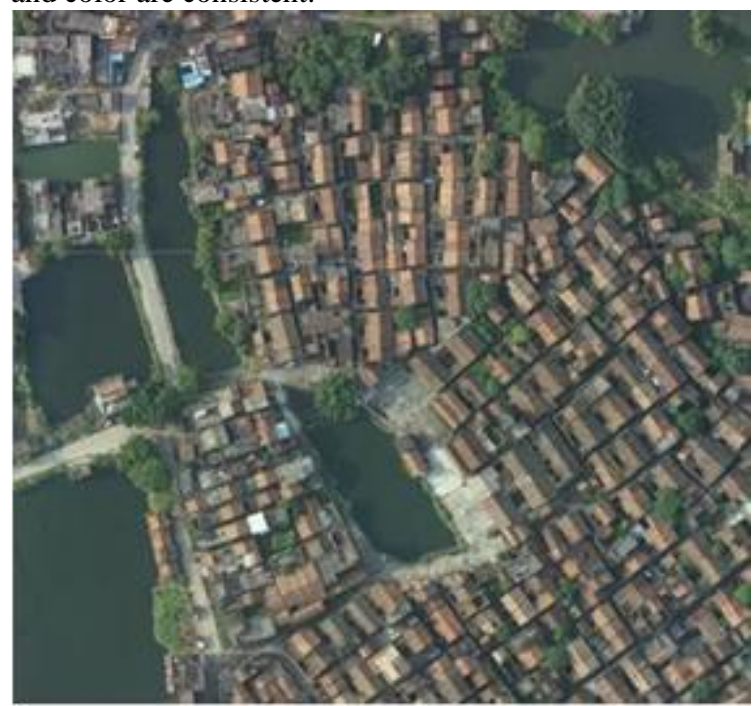

Fig.5 digital orthophoto map

\subsection{Digital Line graph 3D Editing}

The key operational principle of this study is to realize the custom development of the Professional mapping software Map-Matrix platform and the EPS platform. Through the secondary development of EPS platform, the $3 \mathrm{D}$ mapping is the same as the purpose of the GIS data of reached, having a more efficient and advanced DLG production process compared to the majority of the domestic. The main steps are as follows.

A. Data preparation. Prepare the INPHO Aerial triangulation project file and the original photograph data of UAV, put the necessary files into the same folder under the same level directory, such as image ID, external location file, encrypted point file, etc. Keep the file prefix name consistent.

B. New project, open the EPS platform basic mapping platform, select the aerial surveying and editing module, the establishment of local City Geographic template -500 project files.

C. Model recovery, select the 3D map menu, load the stereo image pairs, and restore the 3D model.

D. Stereo editing, articulated external input device, set the work area, start the data collection work.

According to the principle that can actually be observed by the 3D model, the whole factor acquisition should be collected., the observable features can be digitally tracked based on the principle of "mapping positioning and surveying qualification" as far as possible. In this paper, Data for 3D editing all DLG data, a total of 40 pieces of 1: 500 topographic maps were collected and edited.

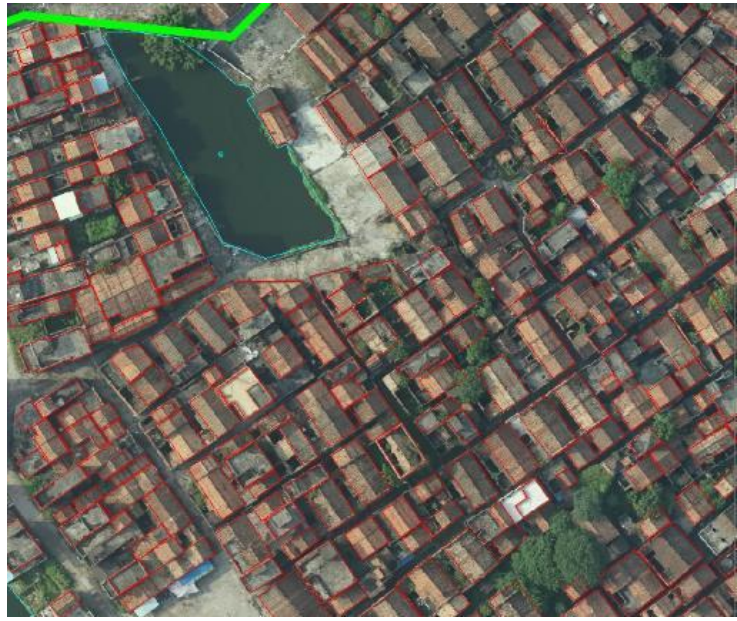

Fig.6 Stereo editing DLG

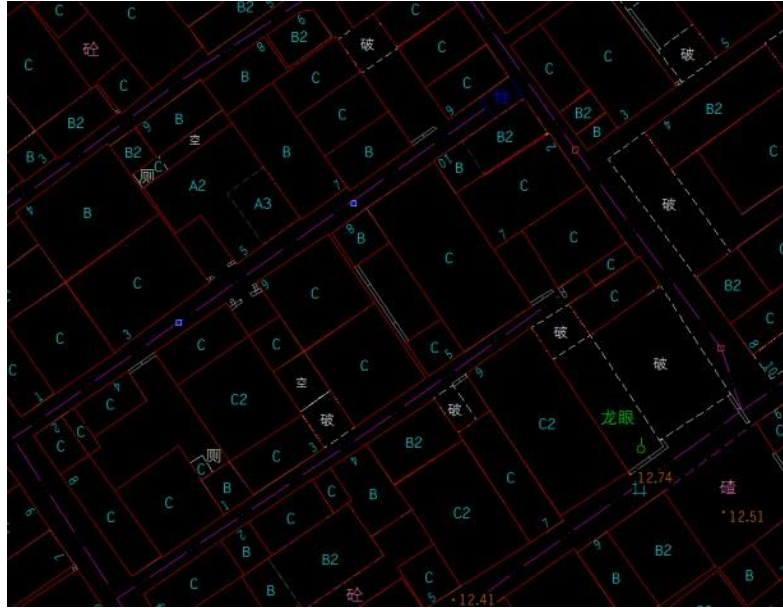

Fig.7 1:500 DLG Partial magnification

\section{KEY TECHNOLOGY OF DATA PROCESSING}

\subsection{Building projection correction}

The DOM is based on the DEM. Since the DOM does not completely eliminate the building projection difference, the position with building projection difference is not correspond to the elevation, thus it can't get the real coordinates of the corresponding objects $^{[11]}$.

Based on the previous research results, this paper uses a method of combining the azimuth elements of the image and the three- dimensional point cloud data of the object. Taking advantage of the collinear relationship of the correspondence points in the projection center, DSM, DEM, a digital elevation 
model is constructed by the elevation difference between DEM image points calculated by DSM object points using the external azimuth element. The projection height difference model following the synchronization change of the image point is formed. Here it is called the projection digital difference model (image elevation synchronous model IESM). The semi- automatic projection of the building correction is realized through the three-dimensional coordinates of the housing corner solved by collinear equation.

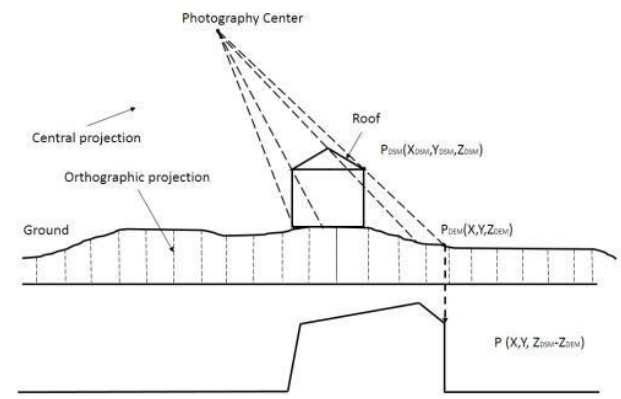

Fig.8 Projection correction principle

\subsection{EPS platform secondary development}

The project mapping work is based on the EPS mapping platform, and uses $\mathrm{VC}++6.0$ development platform and VBScript scripting language to carry out two development of functional expertise. The application layer and the global mathematical function library and control library dynamic link SDL (Sunway Dynamic linked library) are realized, and a platform based on the EPS mapping system is quickly established. The compiled SDL dynamic link library is directly copied to the EPS main directory, achieving dynamic loading or input command to load. The newly developed EPS mapping function integrated modular management through the menu toolbar to load the function, continuing the original functional interface and operating habits of the EPS. At the same time, the data module is standardized to realize 1:500-1: 2000 data standard in the process of mapping display mandatory so as to achieve the four consistency of data production, mapping output, data storage, data application and guarantee data free from alienation effectively.

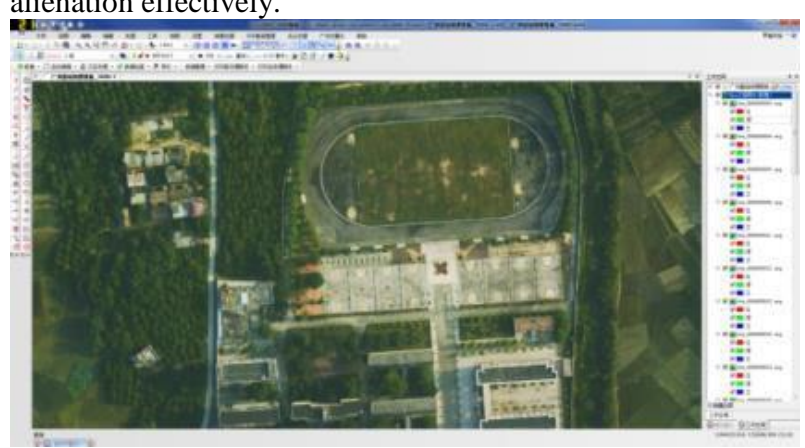

Fig.9 EPS platform for the development of mapping system
**CORS- RTK surveying. 122 plane coordinate and elevation detection points are measured in the whole area. The Relative Mean Square Error (REMS) were calculated by comparing the coordinates of the topographic map with the measured coordinates. According to the results available, this accuracy of aerial mapping of the 1: 500 topographic maps can meet the demands of "Specifications for low- altitude digital aerial photography".

Tab.3 RMS accuracy statistics

\begin{tabular}{|c|c|c|c|c|c|c|c|c|c|c|c|}
\hline \multirow{3}{*}{$\frac{\begin{array}{c}\text { detection } \\
\text { catagory }\end{array}}{\text { point }}$} & \multicolumn{10}{|c|}{ Section } & \multirow{3}{*}{$\begin{array}{c}\text { Total } \\
122\end{array}$} \\
\hline & \multicolumn{2}{|c|}{$0 \sim 1 / 3 \Delta$} & \multicolumn{2}{|c|}{$1 / 3 \Delta \sim 2 / 3 \Delta$} & \multicolumn{2}{|c|}{$2 / 3 \Delta \sim \Delta$} & \multirow{2}{*}{\begin{tabular}{|c|} 
gross \\
2
\end{tabular}} & \multicolumn{3}{|c|}{$\leqslant \Delta / 2$} & \\
\hline & 75 & $61.5 \%$ & 41 & $33.6 \%$ & 4 & $3.3 \%$ & & $1.6 \%$ & 103 & $84.4 \%$ & \\
\hline range & 58 & $69.9 \%$ & 16 & $19.3 \%$ & 6 & $7.2 \%$ & 3 & $3.6 \%$ & 68 & $81.9 \%$ & 83 \\
\hline elevation & 83 & $68.0 \%$ & 26 & $21.3 \%$ & 8 & $6.6 \%$ & 5 & 4. $1 \%$ & 97 & $79.5 \%$ & 122 \\
\hline \multirow{3}{*}{ RIIS } & \multicolumn{3}{|c|}{ point of RUS } & \pm & 10.6 & $\mathrm{~cm}$ & \multicolumn{2}{|c|}{$\Delta$ point } & \pm & 42 & $\mathrm{~cm}$ \\
\hline & \multicolumn{3}{|c|}{ range of RUIS } & \pm & 9.3 & $\mathrm{~cm}$ & \multicolumn{2}{|c|}{$\Delta$ range } & \pm & 20 & $\mathrm{~cm}$ \\
\hline & \multicolumn{3}{|c|}{ elevation of RNS } & \pm & 12.0 & $\mathrm{~cm}$ & \multicolumn{2}{|c|}{$\Delta$ elevation } & \pm & 42 & $\mathrm{~cm}$ \\
\hline
\end{tabular}

\section{CONCLUSION}

In this paper, the production and application of the large scale topographic map for the aerial mapping of the micro unmanned aerial vehicle (UAV) is used in areas of simple terrain and relatively flat terrain, and the results meet the accuracy requirements though field test. The operation efficiency is also greatly improved compared with the traditional measurement model, which can effectively improve the operation efficiency, shorten the production cycle of aerial mapping, and accelerate the integration of internal and external data production process. It also meets the requirement of rapid completion of Surveying and mapping tasks, and provides a new technical means for rapid mapping of large scale topographic maps.

With the accelerating process of urbanization, the demand for large-scale topographic maps is increasing gradually, and the update cycle is getting shorter and shorter. UAV aerial system, with characteristics such as convenient, flexible, high definition, low cost, can greatly reduce the workload of the field and improve data collection efficiency. With the support of the fully automated data processing software, a wealth of results data can be provided in addition to the routine mapping production data results. It has obvious technological advantages in rapid acquisition of large scale topographic maps in traditional surveying difficult areas. With the development of the UAV aerial technology, endurance, flight stability, small frame and other shortcomings will be gradually improved, thus having a broader application prospects and advantages.

\section{RESULTS ACCURACY ANALYSIS}

Based on UAV aerial data, the result of DOM, DEM, DLG etc. were obtained by the above process. In order to ensure the accuracy of the results, 3D coordinate data of external inspection point is quickly obtained by 


\section{REFERENCES}

1. LIU Yujie, CUI Tiejun, GUO Jifa etc. Analysis of Key technologizes of unmanned aerial large scale mapping [J].Journal of Tianjin Normal University (Natural Science Edition) , 2014, 34 (2) : 3740.

2. HE Jing, LI Yongshu, XU Jinghua,etc. Experiment Research of Producing Large-scale Topographic Maps Based on UAV Imagery Data [J].Bulletin Surveying and Mapping, 2009, (8):24-27.

3. CHEN Jie, TONG Xiaohua, LIU Xiangfeng, etc. Photogrammetric Processing of Unmanned Aerial Vehicle Imagery in Heihe Middle Reaches [J]. Geomatics World, 2014, 21(1) : 63-67.

4. TOUTIN T, CHENIER R.GCP Requirement for High resolution Satellite Mapping [J]. International Archives of Photogrammetry, Remote Sensing and Spatial Information Science, 2004, 35(3):836-839.

5. YANG Ailing, YU Hongwei, ZHENG Canhui. Quality Discussion on the Aerial Images of Light UAV [J]. Geomatics \& Spatial Information Technology, 2011, 34(2) : 185-187.

6. LI Yongshu. Research of Drawing the Topographic Map Based on UAV Images [J]. Surveying and Mapping, 2011, 34 (4) : 147-151.
7. XUE Yong'an, WANG xiaoli, ZHANG Mingmei. Fast Survey and Mapping of Large-Scale Topographic Map of Coal Mine Using the UAV Aerial System [J]. journal of Geomatics, 2013, 38(2) : 46-48.

8. ZHANG Huijun. Test and analysis of aerial surveying zonal topographic map using unmanned aerial vehicle $[\mathrm{J}]$. Science of Surveying and Mapping, 2013, 38(3): 100-102.

9. WANG Xiangwen, YU Qisheng, WANG Yapeng. Application of UAV Low-Altitude Photographic System to Large

Scale Topographic Map [J]. Surveying and Mapping of Geology and Mineral Resources, 2013, 29(1) : 34-36.

10. TANG Min,LI Yongshu,LU Heng. Research on the Three-dimensional terrain building Method based on UAV images[C].Proceedings of 2011 International Symposium - Geospatial Information Technology \& Disaster Prevention and Reduction, Chengdu china,May 2011:53-57.

11. LI Changhui, WANG Feng, WANG Lei, etc. Research and Application on Key Technology of Urban Airborne LiDAR Production Technology System [J]. Bulletin Surveying and Mapping, 2013, (8):36-39. 